\title{
Analysis of Serial Search Based Code Acquisition in the Multiple Transmit Antenna Aided DS-CDMA Downlink
}

\author{
SeungHwan Won and Lajos Hanzo \\ School of Electronics and Computer Science, University of Southampton, SO17 1BJ, UK \\ Tel: +44-23-80-593 125, Fax: +44-23-80-594 508

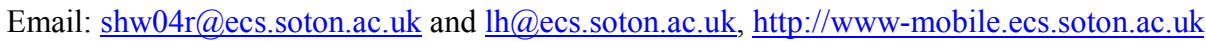

\begin{abstract}
In this contribution we investigate the serial search based initial code acquisition performance of DSCDMA employing multiple transmit antennas both with and without Post-Detection Integration (PDI), when communicating over uncorrelated Rayleigh channels. We characterise the associated performance trends as a function of the number of transmit antennas. It is demonstrated that in contrast to our expectation, the achievable correct detection probability $P_{D}$ degrades at low $E_{c} / I_{o}$ values, as the number of transmit antennas is increased. It is extremely undesirable to degrade the achievable acquisition performance, when the system is capable of attaining its target bit error rate performance at reduced SINR values, as a benefit of employing multiple transmit antennas. Our future research will focus on the study of designing iterative turbo-like acquisition schemes designed for MIMO systems.
\end{abstract}

\section{INTRODUCTION}

The application of multiple antennas in the downlink of wireless systems constitutes an attractive technique of reducing the detrimental effects of time-variant multipath fading environments [1,2]. In inter-cell synchronous CDMA systems the mobile station's (MS) receiver must be capable of synchronising a locally generated pseudonoise (PN) code with the received multi-user signals containing the desired user's PN sequence. Substantial research efforts have been devoted to the design of code acquisition techniques [3]. However, most of them have been designed for Single-Input SingleOutput (SISO) systems [4-6]. Hence there is a paucity of code acquisition techniques designed for transmit diversity aided systems [7]. A variety of serial search [46], parallel search [8], as well as sequential estimation $[9,10]$ based code acquisition techniques have been proposed in literature.

The classic serial search technique has been employed in scenarios, where the uncertainty region (or search window width) is quite wide and hence in the context of serial search the mean acquisition time is the vital performance criterion, as seen in the downlink of the inter-cell synchronous CDMA-2000 system [4,5]. Against this background, in this treatise we investigate a serial search based technique designed for multiple

The financial support of the Ministry of Information and Communication(MIC), Republic of Korea and the European Union under the auspices of the Phoenix and Newcom projects and that of the EPSRC is gratefully acknowledged. transmit antenna aided systems. More explicitly, we quantify both the correct detection probability as well as the false alarm probability as a function of the Signal-toInterference plus Noise Ratio (SINR) per chip $\left(E_{c} / I_{o}\right)$, and that of the number of transmit antennas both with and without PDI.

This paper is organised as follows. Section 2 describes the system investigated, while the correct detection and false alarm probability are analysed in the context of uncorrelated Rayleigh channels in Section 3. In Section 4, our numerical results are discussed and our conclusions are offered in Section 5.

\section{SYSTEM DESCRIPTION}

The transmitted signal at the $n^{\text {th }}$ chip sampling instant of the $k^{\text {th }}$ user at the base station of a multiple transmit antenna aided system can be expressed as

$x_{n}(k) a_{n}(k) w_{n, p}(k) \sqrt{\frac{E_{c}(k)}{P}} h\left(t-n T_{c}\right) \sqrt{2} \cos \left(2 \pi f_{0} t+\phi_{k}\right),(1)$ where $p=1, \ldots, P$ is the number of transmit antennas, $x_{n}(k)$ represents the binary input data sequence assuming values of +1 or $-1, a_{n}(k)$ denotes the $k^{\text {th }}$ user's unique user-specific PN sequence, $w_{n, p}(k)$ identifies the specific Walsh code assigned to the $p^{\text {th }}$ transmit antenna, $E_{c}$ denotes the pilot signal energy per PN code chip, $h(t)$ is the impulse response of the pulse shaping filter, $T_{c}$ is the chip duration, $f_{0}$ is the carrier frequency and $\phi_{k}$ is the carrier phase of the $k^{\text {th }}$ user's modulator. The total allocated power is equally shared by the $P$ transmit antennas.

Fig. 1 portrays a transmit diversity aided CDMA system. The tapped-delay line channel model generates $q$ multi-path signals arriving with a time delay of $\tau_{i}[5,6]$. Therefore, the received signal $r_{k}$ is a composite of $q$ multi-path signals having a time delay of $\tau_{i}$ expressed in the form of

$$
\begin{gathered}
r_{k}=\sum_{i=1}^{q} \sum_{j=1}^{P} \alpha_{i, j} \sqrt{\frac{E_{c}(k)}{P}} x_{n}(k) a_{n}(k) w_{n, j}(k) h\left(t-n T_{c}-\tau_{i}\right) \\
\cdot \sqrt{2} \cos \left(2 \pi f_{0} t+\phi_{k, i}\right),
\end{gathered}
$$

where $\alpha_{i, j}$ represents the envelope of the $i^{\text {th }}$ path signal obeying the Rayleigh distribution. Furthermore, $\phi_{k, i}$ denotes the signal phase of $i^{\text {th }}$ path having a uniform distribution over $(0,2 \pi)$. In the case of the initial synchronisation of DS-CDMA, the main goal is to acquire perfect timing of the first arrived path at the 
receiver, because this timing information is used as that of the reference finger of the Rake receiver. Therefore we focus our attention on the performance analysis of the first received path and consider the multi-path interference effects within the reference cell.

Fig 2 depicts the block diagram of the noncoherent receiver designed for our code acquisition scheme using both multiple transmit antennas and PDI, which generates a decision variable by accumulating $L$ consecutive signals observed over multiple time intervals to improve the correct detection probability in the mobile channel imposing both fading and poor SINR conditions $[4,5,7]$. In this figure, following the procedures outlined in [4,5], the mean values of the Iand Q- channel outputs seen in Fig.2, are given by

$$
\begin{aligned}
& E\left[Y_{\text {tot }}^{(I)}(k)\right]=\sum_{i=1}^{P} \alpha_{i} N \sqrt{\frac{E_{c}(k)}{P}} R(\tau) \cos \phi_{k}, \\
& E\left[Y_{\text {tot }}^{(q)}(k)\right]=\sum_{i=1}^{P} \alpha_{i} N \sqrt{\frac{E_{c}(k)}{P}} R(\tau) \sin \phi_{k},
\end{aligned}
$$

where the autocorrelation function of the timing error is given by $R(\tau)=\int_{-\infty}^{\infty}\left|H(f)^{2}\right| \cos (2 \pi f \tau) d f$. For simplicity, $R(\tau)$ is considered to be 1 .

\section{CORRECT DETECTION AND FALSE ALARM PROBABILITY}

For the sake of deriving the likelihood functions conditioned on both the hypothesis of the desired signal being present and absent, we introduce the likelihood functions of $Y^{(I)}$ and $Y^{(Q)}$, which may be expressed in the context of an AWGN channel [4] as

$$
\begin{aligned}
& P_{0}\left(Y^{(I)}, Y^{(Q)}\right)=\exp \left[-\left(\left(Y^{(I)}\right)^{2}+\left(Y^{(Q)}\right)^{2}\right) / V\right] / \pi V, \\
& P_{1}\left(Y^{(I)}, Y^{(Q)} \mid \phi\right)=\exp \left[-\left(Y^{(I)}-N \sqrt{\frac{E_{c}}{P}} \cos \phi\right)^{2} / V\right] \\
& \cdot \exp \left[-\left(Y^{(Q)}-N \sqrt{\frac{E_{c}}{P}} \sin \phi\right)^{2} / V\right] / \pi V .
\end{aligned}
$$

The variances of $Y^{(I)}$ and $Y^{(Q)}$ are given by $V_{I}=V_{Q}=\frac{V}{2}$, where we have $V=N I_{0}$. The total amount of channel-induced impairments in the downlink is constituted by the superposition of the background noise, plus the serving-cell interference imposed by the multi-path signals and the other-cell interference. Further details on the calculation of the total interference may be found in $[4,5]$. Since the carrier phase is modelled by a uniform random variable, we represent the unconditional likelihood function in the presence of the desired signal by averaging $\operatorname{Eq}(6)$ over $\phi$, yielding

$$
\begin{aligned}
& P_{1}\left(Y^{(I)}, Y^{(Q)}\right)=\int_{0}^{2 \pi} P_{1}\left(Y^{(I)}, Y^{(Q)} \mid \phi\right) d \phi \frac{1}{2 \pi} \\
= & \int_{0}^{2 \pi} \exp \left\{-\left[\left(Y^{(I)}\right)^{2}+\left(Y^{(Q)}\right)^{2}+\frac{N^{2} E_{c}}{P}\right] / V\right\} \\
& \frac{\exp \left\{2 N \sqrt{\frac{E_{c}}{P}}\left[Y^{(I)} \cos \phi+Y^{(Q)} \sin \phi\right] / V\right\}}{\pi V} \frac{d \phi}{2 \pi} \\
= & \frac{1}{\pi V} \exp \left(\frac{-\left[\left(Y^{(I)}\right)^{2}+\left(Y^{(Q)}\right)^{2}\right]}{V}\right)
\end{aligned}
$$

$$
I_{0}\left(\frac{2 \sqrt{\frac{N^{2} E_{c}}{P}\left[\left(Y^{(I)}\right)^{2}+\left(Y^{(Q)}\right)^{2}\right]}}{V}\right) \exp \left(-\frac{N^{2} E_{c}}{P} / V\right),
$$

where $I_{0}(x) \equiv \int_{0}^{2 \pi} \exp (x \cdot \cos \phi) d \phi / 2 \pi$ is the zeroth-order modified Bessel function.

Then, the mean of the output variable, $Z$, is expressed as $E[Z]=\left(E\left[Y^{(I)}\right]\right)^{2}+\left(E\left[Y^{(Q)}\right]\right)^{2}=N^{2}\left(\frac{E_{c}}{P}\right)$ and the square of the means of $Y^{(I)}$ and $Y^{(Q)}$ is given by $M^{2}=N^{2}\left(\frac{E_{c}}{P}\right)$.

By using the Jacobian transformation [11], we arrive at the likelihood functions conditioned on both the hypothesis of the desired signal being present and absent in the context of an AWGN channel

$$
\begin{aligned}
& p_{0}(Z)=\exp [-Z / V] / V, Z>0, \\
& p_{1}(Z)=\frac{\exp \left[-\left(Z+M^{2}\right) / V\right]}{V} I_{0}\left(\frac{2 \sqrt{M^{2} Z}}{V}\right) .
\end{aligned}
$$

For the sake of expressing the likelihood function conditioned on the presence of the desired signal in $p_{1}(Z)$ derived for transmission over an uncorrelated Rayleigh channel, first the likelihood function $p_{1}(Z, \beta)$ corresponding to the signal-to-noise ratio $\beta$ conditioned on the hypothesis of the desired signal being transmitted over an AWGN channel is weighted by the probability of occurrence $p(\beta)$ of encountering the SNR $\beta$ quantified by the PDF and then averaged over its range of $-\infty \sim \infty$, yielding:

$$
\begin{aligned}
p_{1}(Z) & =\int_{-\infty}^{\infty} p(\beta) p_{1}(Z, \beta) d \beta \\
& =\int_{0}^{\infty} \frac{e^{-\beta / \sigma^{2}}}{\sigma^{2}} \frac{\exp \left[\left(-Z+\beta \mathrm{M}^{2}\right) / V\right]}{V} I_{0}\left(\frac{2 \sqrt{\beta \mathrm{M}^{2} Z}}{V}\right) d \beta \\
& =\frac{\exp \left[-Z /\left(V+M^{2} \sigma^{2}\right)\right]}{V+M^{2} \sigma^{2}} \\
& \equiv \frac{\exp \left[-Z /\left(V+\bar{M}^{2}\right)\right]}{V+\bar{M}^{2}},
\end{aligned}
$$

where we have $\bar{M}^{2} \equiv M^{2} \sigma^{2}=N^{2} \bar{E}_{c}$.

Upon defining $V_{F}=V+\bar{M}^{2}=N\left(I_{0}+N \bar{E}_{c}\right)$, and

$\bar{E}_{c}=\sigma^{2}\left(\frac{E_{c}}{P}\right)$, we arrive at

$p_{0}(Z)=\frac{1}{V} e^{-Z / V}, \quad$ (11) $p_{1}(Z)=\frac{1}{V_{F}} e^{-Z / V_{F}}$.

In this paper we essentially limit ourselves to considering multiple transmit antennas both with and without PDI and a single receive antenna. Nonetheless, we briefly highlight the effects of additionally using multiple receive antennas in conjunction with PDI. Here we assume that the multiple received signals are independent in both the spatial and the time domain, which leads to a straightforward extension of the 
formulas to the more general scenarios of invoking multiple transmit/receive antennas. Since the final decision variable is constituted by the sum of $(P \cdot R \cdot L)$

number of independent variables $\left(Z_{t o t}=\sum_{i=1}^{P} \sum_{j=1}^{R} \sum_{k=1}^{L} Z_{i, j, k}\right)$, each of which has a likelihood function given by (11) or (12), we can determine the Laplace transform of each, by raising them to the $(P \cdot R \cdot L)^{\text {th }}$ power and then carry out the inverse transform for the sake of generating the desired likelihood function [4].

Assuming that the number of transmit antennas is $P$, the number of receive antennas is $R$ and the number of PDI stages is $L$, in the case of multiple transmit/receive antennas using PDI, we arrive at

$$
\begin{aligned}
& p_{0}(Z)=\frac{Z^{P R L-1} e^{-Z / V}}{(P R L-1) ! V^{P R L}}, \\
& p_{1}(Z)=\frac{Z^{P R L-1} e^{-Z / V_{F}}}{(P R L-1) ! V_{F}^{P R L}} .
\end{aligned}
$$

Using the procedures outlined above, the probability of correct detection is obtained as follows:

$$
P_{D}=\int_{\theta}^{\infty} p_{1}(Z) d Z=\exp \left(-\frac{\theta}{V_{F}}\right) \sum_{k=0}^{P R L-1} \frac{\left(\theta / V_{F}\right)^{k}}{k !} .
$$

Similarly, the probability of false alarm is expressed as

$P_{F}=\int_{\theta}^{\infty} p_{0}(Z) d Z=\exp \left(-\frac{\theta}{V}\right) \sum_{k=0}^{P R L-1} \frac{(\theta / V)^{k}}{k !}$,

where $\theta$ is a threshold value. In the following section, we will numerically evaluate the achievable system performance based on the above formulas.

\section{NUMERICAL SYSTEM PERFORMANCE RESULTS}

In this section we will characterise the code acquisition performance of multiple transmit antenna aided CDMA systems both with and without PDI. The system parameters are summarised in Table 1 . In Table 2 we outlined the maximum SINR degradation imposed by both the Doppler shift and the frequency mismatch between the transmitter and receiver in conjunction with a coherent integration interval of $N$ chip duration. It may be deemed sufficient at this point to integrate the detector output seen in Fig. 2 over $N=256$ chips, which is equivalent to two 128-chip modulated symbols used for coherent accumulation. This value was calculated by using the formula of [4,Eq.(3.7)] provided for determining the performance degradation owing to both the Doppler shift and the frequency mismatch. The spreading factor of the Walsh code was selected to be 128. The frequency mismatch was assumed to be $1000 \mathrm{~Hz}$ [4], while the carrier frequency was $900 \mathrm{MHz}$. Finally, as a worst-case mobile speed, it is reasonable to postulate $160 \mathrm{~km} / \mathrm{h}$. We also assumed that the sampling inaccuracy caused by having a finite search step size of $\Delta=1 / 2 T_{C}$ was $-0.91 \mathrm{~dB}$, which is a typical value of the search step size $[4,5]$. Accordingly, we considered three performance degradation factors, which encompassed the frequency mismatch, the Doppler shift and the effects of the finite sampling distance. All these imperfections were taken into account, when calculating the correct detection probability.

Fig.3 illustrates the correct detection versus false alarm probability, parameterised by both the number of transmit antennas for $P=1,2,4$ as well as 10 and the $E_{c} / I_{o}$ ratio. In case of $E_{c} / I_{o}=-10 \mathrm{~dB}$, the achievable performance enhancement gradually saturates, as the transmit diversity order is increased from $P=1$ to 10. By contrast, $P_{D}$ decreases as the number of transmit antennas $P$ increases, when the mobile station experiences a relatively low $E_{c} / I_{o}$ ratio of $-19 \mathrm{~dB}$, as evidenced by the four curves corresponding to lower $P_{D}$ values in Fig.3. Fig.4 characterises the correct detection versus false alarm probability, parameterised by both the number of transmit antennas for $P=1,2$ as well as 4 in conjunction with both $L=2$ (Left figure) and $L=4$ (Right figure) PDI stages and by the $E_{c} / I_{o}$ ratio.

When having $L=2$ PDI stages, as portrayed at the left of Fig.4, the result shows similar trends to those of Fig.3. By contrast, in the scenario of $L=4$ PDI stages characterised at the right of Fig.4, there is a sufficiently high time diversity gain, which has beneficial effects on the achievable acquisition performance $P_{D}$. However, as seen at the right of Fig.4, increasing the transmit diversity order imposes a degradation of the achievable $P_{D}$ performance. These specific $E_{c} / I_{o}$ abscissa values in Fig.3 and Fig.4 were chosen to exemplify the typical achievable values. In all the remaining figures we will assume an operation in the range of 'finger locking', which may be considered to be the range between $E_{c} / I_{o}=-17$ and $-13 \mathrm{~dB}$, as suggested in [12]. Therefore in Fig.5 and Fig.6 we will investigate in more detail the somewhat unexpected phenomenon of experiencing a degraded acquisition performance in the presence of multiple transmit antennas. The correct detection probabilities seen in Fig.5 and Fig.6 were obtained assuming a false locking probability of $P_{F}=0.1$ for all scenarios considered.

In Fig.5 and Fig.6, the relationship between $P_{D}$ and the number of transmit antennas is portrayed both with and without PDI for different values of $E_{c} / I_{o}$, respectively. More explicitly, Fig. 5 portrayed the correct detection probability versus the number of transmit antennas, parameterised by the pilot channel SINR of $E_{c} / I_{o}$. At $E_{c} / I_{o}=-10 \mathrm{~dB}$ a slight $P_{D}$ improvement is observed upon increasing the number of transmit antennas, although again, at low $E_{c} / I_{o}$ values typically the opposite is true. Fig. 6 characterises the correct detection probability versus both the number of transmit antennas and the number of PDI stages, parameterised by the pilot channel's $E_{c} / I_{o}$ value. The left illustration of Fig. 6 characterises the scenario of $L=2$ PDI stages, while the one at the right of Fig.6 was valid for $L=4$ PDI stages. The curve recorded for $E_{c} / I_{o}=-10 \mathrm{~dB}$ at the right of Fig.6, overlapped with that plotted for $E_{c} / I_{o}=-$ $13 \mathrm{~dB}$, because all the achievable correction probabilities were $P_{D}=1$. Both Fig.5 and 6 illustrate that $P_{D}$ tends to decrease, as the number of transmit antennas increases, especially when the mobile station experiences a low $E_{c} / I_{o}$ value. We can observe in both Fig.5 and Fig.6 that the highest detection probabilities marked by circles 
were achieved, when the per-branch $E_{c} / I_{o}$ value was $19 \mathrm{~dB}$ for a given total $E_{c} / I_{o}$ value in the range of 'finger locking', except for the scenarios of $L=4$ PDI stages. Owing to the above-mentioned facts, the range of the minimum $E_{c} / I_{o}$ values required for reaching 'finger locking' may vary, depending upon the number of transmit antennas.

The techniques associated with the multiple transmit antennas constitute an efficient means of improving the attainable diversity gain, when communicating over mobile channels. However, for the sake of fair comparisons the total signal power transmitted from the multiple transmit antennas must be fixed, regardless of the number of transmit antennas. In other words, the total transmit power must be equally shared by all the transmit antennas. This implies that an excessively low level of the per-branch received signal strength would lead to a low acquisition performance, even if the transmit diversity gain is high. In other words, a high diversity order effectively results in an acquisition performance loss, as a consequence of the insufficiently high transmit signal strength per branch. In the case of employing both multiple transmit antennas and PDI, this trend is still observable, although using two or four PDI stages has the potential of mitigating the associated acquisition performance degradation imposed by the low per-branch $E_{c} / I_{o}$ values encountered.

\section{CONCLUSION}

In this paper, we analysed the multiple antenna aided transmit diversity effects on the initial acquisition performance of the inter-cell synchronous CDMA downlink. The probabilities of correct detection and false alarm have been derived analytically and numerical results were provided. Ironically, our findings suggest that increasing the number of transmit antennas results in combining the low-energy, noise-contaminated signals of the transmit antennas, which ultimately reduces the correct detection probability. However, it is extremely undesirable to degrade the achievable acquisition performance, when the system is capable of attaining its target bit error rate performance at reduced SINR values, as a benefit of employing multiple transmit antennas. It may be concluded that the achievable cell coverage determined by the received pilot channel power may be reduced, as the number of transmit antennas is increased, which is a highly undesirable phenomenon, since it has grave repercussions in terms of having to tolerate a high number of handovers per cell. Our future research will focus on the study of designing iterative turbo-like acquisition schemes $[3,10]$ designed for MIMO systems.

\section{REFERENCES}

[1] D. Gesbert, M. Shafi, D.S. Shiu, P.J. Smith, and A. Naguib, From Theory to Practice: An Overview of MIMO Space-Time Coded Wireless Systems, pp281-302, IEEE Journal on Selected Areas in Communications, vol. 21, NO.3, Issue 3, 2003.

[2] R.A. Soni, R.M. Buehrer, On the Performance of Open-Loop Transmit Diversity Techniques for IS2000 Systems: A Comparative Study, pp1602--1615, IEEE Transactions on Wireless Communications, vol. 3, NO.5, Issue 5, 2004.
[3] L. Hanzo, L-L Yang, E-L Kuan, K. Yen, Single- and Multi- Carrier DS-CDMA, IV Multi-Carrier CDMA, Chapter 21, Initial Synchronization of DS-CDMA and MC-CDMA Systems, Wiley, 2003.

[4] A.J. Viterbi, CDMA: Principles of Spread Spectrum Communication, Chapter 2 and 3, Addison-Wesley, 1995.

[5] H.R. Park, Performance Analysis of a Double-Dwell Serial Search Technique for Cellular CDMA Networks in the Case of Multiple Pilot Signals, pp1819--1830, IEEE Transactions on Vehicular Technology, vol. 48, NO.6, Issue 6, 1999.

[6] L-L Yang, L. Hanzo, Serial Acquisition of DSCDMA Signals in Multipath Fading Mobile Channels, pp617--628, IEEE Transactions on Vehicular Technology, vol. 50, NO.2, Issue 2, 2001.

[7] S.H. Won and Y.J. Kim, Performance Analysis of Multi-path Searcher for Mobile Station in W-CDMA System Employing Transmit Diversity, pp137--139, Electronics Letters, vol. 39, Issue 1, 2003.

[8] K.K. Chawla and D.V. Sarwate, Parallel acquisition of PN sequences in DS/SS systems, pp2155--2164, IEEE Transactions on Communications, vol. 42, Issue $5,1994$.

[9] R.B. Ward, Acquisition of pseudonoise signals by sequential estimation, pp475--483, IEEE Transactions on Communications Technology, vol. 13, Issue 4, 1965

[10] L-L Yang, L. Hanzo, Acquisition of m-sequences using recursive soft sequential estimation, pp199--204, IEEE Transactions on Communications, vol. 52, Issue 2, 2004.

[11] A. Leon-Garcia, Probability and Random Processes for Electrical Engineering: $2^{\text {nd }}$ edition, Chapter 4, Multiple Random Variables, Addison-Wiley, 1994.

[12] C. Noblet, M. Fadridis and R. Owen, Downlink Transmit Power Issues in a WCDMA Cellular System, 3G Mobile Communication Technologies, 2002 Conference Publication No. 489, pp244--249, 8-10 May 2002.

Table1. System Parameters

\begin{tabular}{|c|c|c|}
\hline \multicolumn{2}{|l|}{ Bandwidth } & $1.2288 \mathrm{MHz}$ \\
\hline \multicolumn{2}{|c|}{ Carrier frequency } & $900 \mathrm{MHz}$ \\
\hline \multicolumn{2}{|c|}{ Spreading factor } & 128 \\
\hline \multirow[t]{2}{*}{ Diversity: } & Transmit & $1,2,4,6,8,10$ \\
\hline & PDI & $1,2,4$ \\
\hline \multicolumn{2}{|c|}{ Frequency mismatch } & $1000 \mathrm{~Hz}$ \\
\hline \multicolumn{2}{|c|}{ Mobile speed } & $160 \mathrm{~km} / \mathrm{h}$ \\
\hline \multicolumn{2}{|c|}{ Coherent integration interval } & 256chips \\
\hline
\end{tabular}

Table2. Maximum SINR degradation inflicted by both the Doppler shift and a $1000 \mathrm{~Hz}$ frequency mismatch in conjunction with the coherent integration interval of $\mathrm{N}$ chip durations at a carrier frequency of $900 \mathrm{MHz}$

\begin{tabular}{|c|c|c|c|c|c|}
\hline $\mathrm{N}$ (Chips) & 64 & 128 & 256 & 384 & 512 \\
\hline $\begin{array}{c}\text { Degradation } \\
(\mathrm{dB})\end{array}$ & 0.0487 & 0.1954 & 0.7923 & 1.8267 & 3.3708 \\
\hline
\end{tabular}




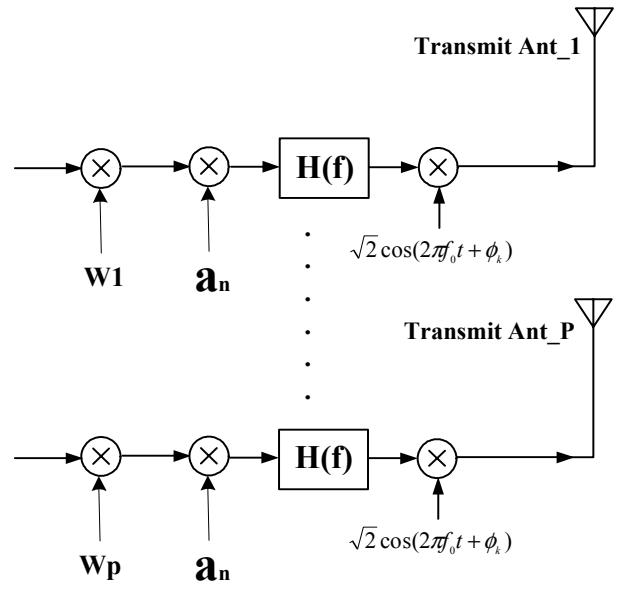

Figure1. Downlink Transmit Diversity System Structure.

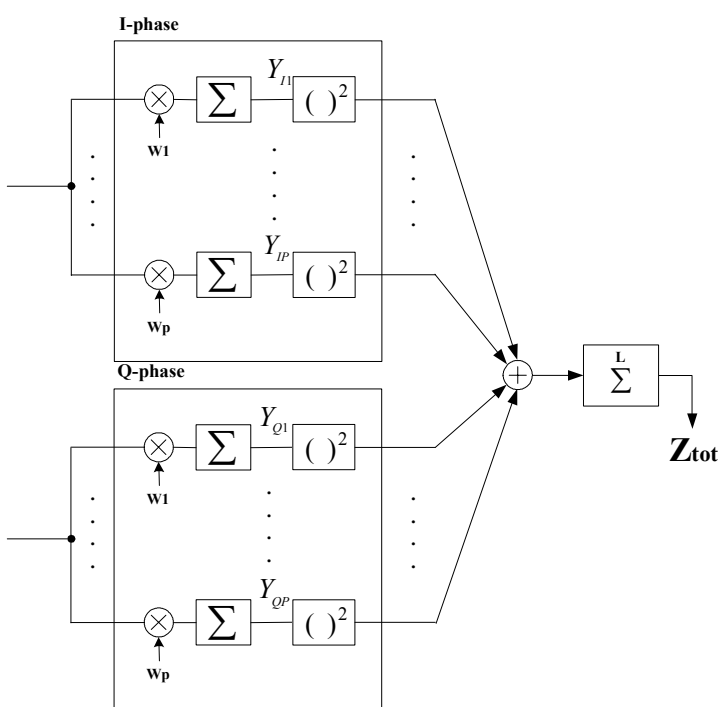

Figure2. Receiver Structure.

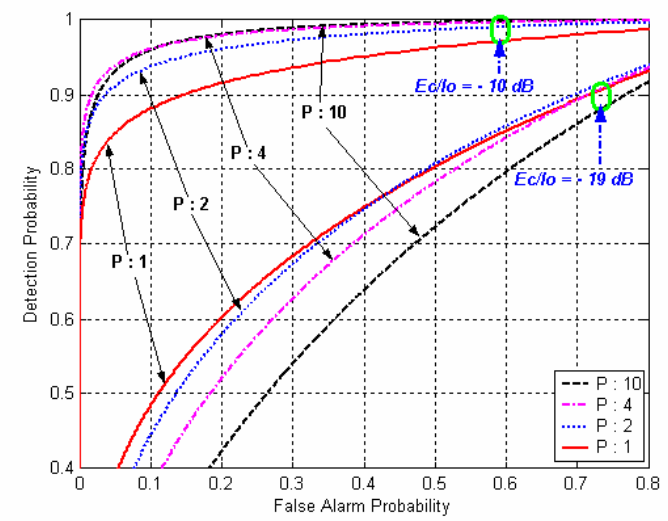

Figure3. Correct detection versus false alarm probability for $P=1,2,4$, and 10 transmit antennas for transmission over uncorrelated Rayleigh channels, where the lines indicate the theoretical values evaluated from Eqs(15) and (16).
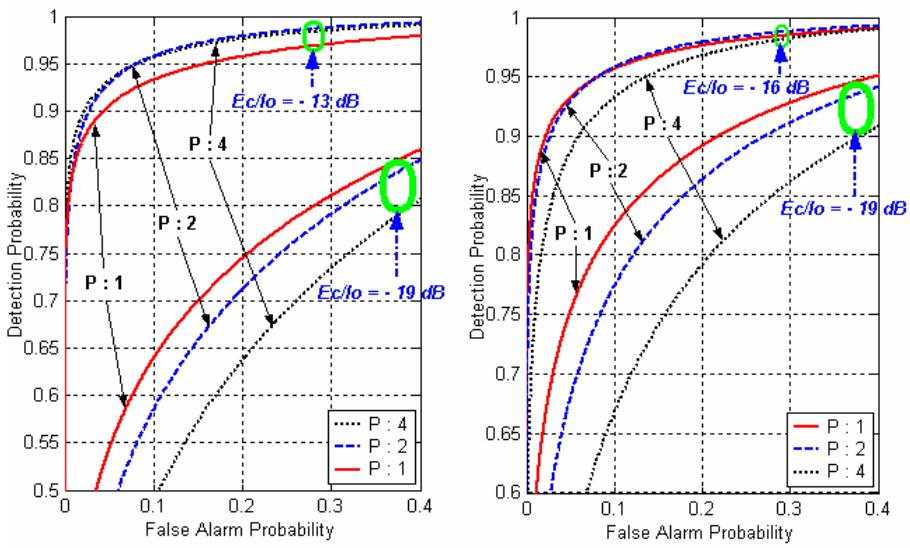

Figure4. Correct detection versus false alarm probability for $P=1,2$ as well as 4 transmit antennas in conjunction with both $L=2$ (Left figure) and $L=4$ (Right figure) PDI stages for transmission over uncorrelated Rayleigh channels, where the lines indicate the theoretical values evaluated from Eqs(15) and (16).

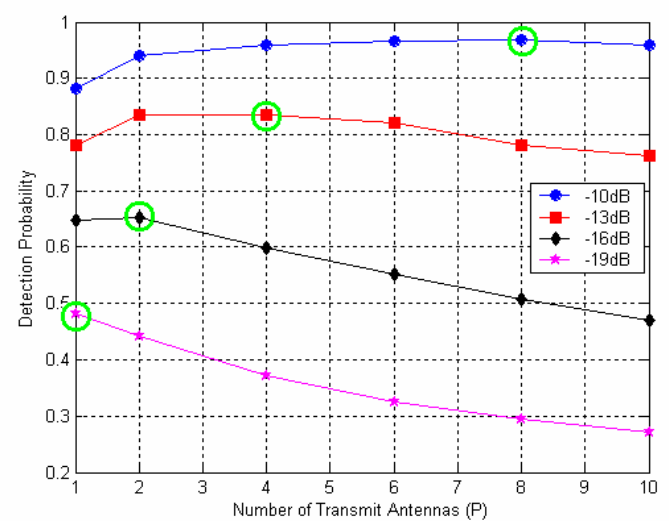

Figure5. Correct detection probability versus the number of transmit antennas for $P=1,2,4,6,8$ and 10, parameterised by the pilot channel 'SINR per chip denoted as $E_{c} / I_{o}$ ' for transmission over uncorrelated Rayleigh channels.
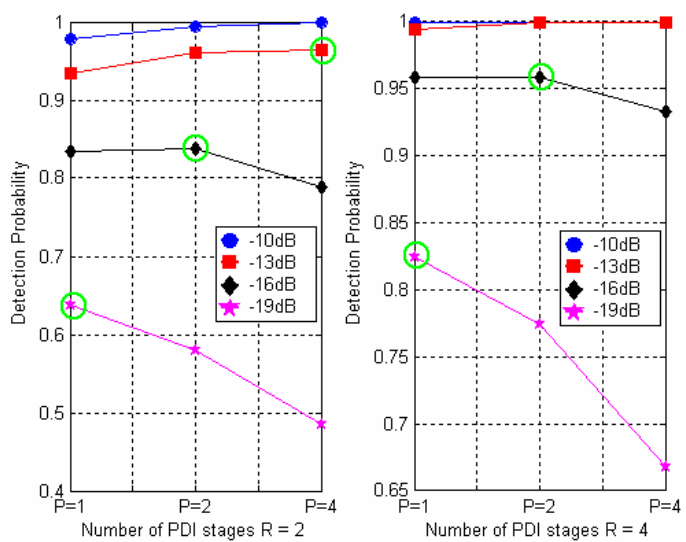

Figure6. Correct detection probability versus the number of transmit antennas for $P=1,2$ as well as 4 and the number of PDI stages for both $L=2$ (Left figure) and $L=4$ (Right figure), parameterised by the pilot channel 'SINR per chip denoted as $E_{c} / I_{o}$ ' for transmission over uncorrelated Rayleigh channels. 